\title{
Mars Dust Storms: Interannual Variability and Chaos
}

\author{
ANDREW P. INGERSOLL AND JAMES R. LyONS \\ Division of Geological and Planetary Sciences, California Institute of Technology, Pasadena

\begin{abstract}
The hypothesis is that the global climate system, consisting of atmospheric dust interacting with the circulation, produces its own interannual variability when forced at the annual frequency. The model has two time-dependent variables representing the amount of atmospheric dust in the northern and southern hemispheres, respectively. Absorption of sunlight by the dust drives a cross-equatorial Hadley cell that brings more dust into the heated hemisphere. The circulation decays when the dust storm covers the globe. Interannual variability manifests itself either as a periodic solution in which the period is a multiple of the Martian year, or as an aperiodic (chaotic) solution that never repeats. Both kinds of solution are found in the model, lending support to the idea that interannual variability is an intrinsic property of the global climate system. The next step is to develop a hierarchy of dust-circulation models capable of being integrated for many years.
\end{abstract}

\section{INTRODUCTION}

Interannual variability is perhaps the most puzzling aspect of Martian global dust storms. It is not enough to explain how dust storms work; one must also explain why they do not work every year. On Mars there are no obvious longterm variables like oceanic heat storage, seasonal snow cover, sea ice, or vegetation that might fluctuate from year to year and provide some basis for long-term variability. The longest natural time constant, about $1 / 10$ the Martian year, is associated with the fallout of dust from the atmosphere. One can invoke hidden microphysical variables, like the placement of dust grains in relation to surface roughness elements, or one can assume that the Martian climate is in such a delicate balance that even small random fluctuations from one year to the next determine whether a global dust storm occurs. But it is also possible that the global distribution of atmospheric dust, with its attendant effects on the global circulation, provides enough memory so that the state of the system one year determines the initial conditions for the next. Toss in some nonlinear interaction among the variables, add seasonal forcing, and one may have a recipe for chaos. That is the hypothesis of this paper.

Martin and Zurek have summarized the observations of Martian global dust storms [Martin, 1974a, b, 1976, 1984; Zurek, 1982; Zurek and Martin, 1993]. Zurek and Martin [1993] use the phrase "planet encircling" to describe the six large storms that were observed. Although good observations were made in 1905-1911, 1922-1926, 1937-1943, and 1986-1991, no planet-encircling storms were seen during these years. The first planet-encircling storm seen from Earth was in 1956. Other such storms were seen in 1971 and 1973. Two storms in 1977 and one in 1982 were not seen from Earth but were detected by the Viking instruments. Zurek and Martin estimate that the chance of a planet-encircling storm occurring in any arbitrary Mars year is $\leq 50 \%$, if such occurrence is random by year and yet restricted seasonally to southern spring and summer.

The seasonality of the planet-encircling dust storms is an important feature. The six that were observed all occurred during southern spring and summer. They develop in the

Copyright 1993 by the American Geophysical Union.

Paper number 93JE00676.

0148-0227/93/93JE-00676\$05.00 southern subtropics and spread into the northern hemisphere in 10-20 days [Martin, 1974a, $b, 1976]$. Southern summer solstice $\left(L s=270^{\circ}\right)$ occurs just after perihelion passage ( $L s$ $\approx 250^{\circ}$, where $L s$ is the angle of Mars in its orbit from spring equinox), so most theories of the dust storms have focused on the extra amount of sunlight incident at this time (the orbital eccentricity $\varepsilon$ is 0.093 ). Interannual variability is a separate issue that has not been addressed in a quantitative way.

Theories of Martian global dust storms have been described by a number of authors [Gierasch and Goody, 1973; Leovy et al., 1973, 1985; Gierasch, 1974; Pollack et al., 1979; Schneider, 1983; Haberle, 1986; Zurek et al., 1992]. Gierasch and Goody [1973] developed the idea of a dust-driven circulation, whereby absorption of sunlight by the dust sets up horizontal temperature gradients that intensify the winds and bring in more dust. They were thinking of a dusty hurricane, but the idea applies as well to the cross-equatorial Hadley cell that intensifies itself by picking up dust and bringing it into the summer hemisphere. There is abundant evidence that dust heats the atmosphere. Both two- and three-dimensional numerical models have demonstrated that solar heating of airborne dust provides positive feedback leading to a stronger and more extensive meridional circulation [e.g., Haberle et al., 1982, 1993; Schneider, 1983; Pollack et al., 1990; Zurek et al., 1992; Murphy et al., 1993]. Viking Lander data revealed much about the interaction between dust storms and baroclinic wave activity in the northern winter hemisphere. These data help define the two regimes that can occur during this season, one with global dust storms and one without [Leovy et al., 1985; Haberle, 1986], but they do not explain the occurrence of one regime in some northern winters and the other regime in other northern winters. The problem of interannual variability remains.

Our hypothesis is that the global climate system, without exotic microphysical processes or delicate balances, is the source of its own interannual variability. We use the circulation-dust feedback idea and apply it to the cross-equatorial Hadley cell, but realism is not the primary goal. Rather, we ask the question whether such a system, whose natural time constant is the lifetime of a global dust storm, can exhibit interannual variability when forced at the annual frequency. We assume that the dust storm lifetime is $1 / 10$ of the Mars 


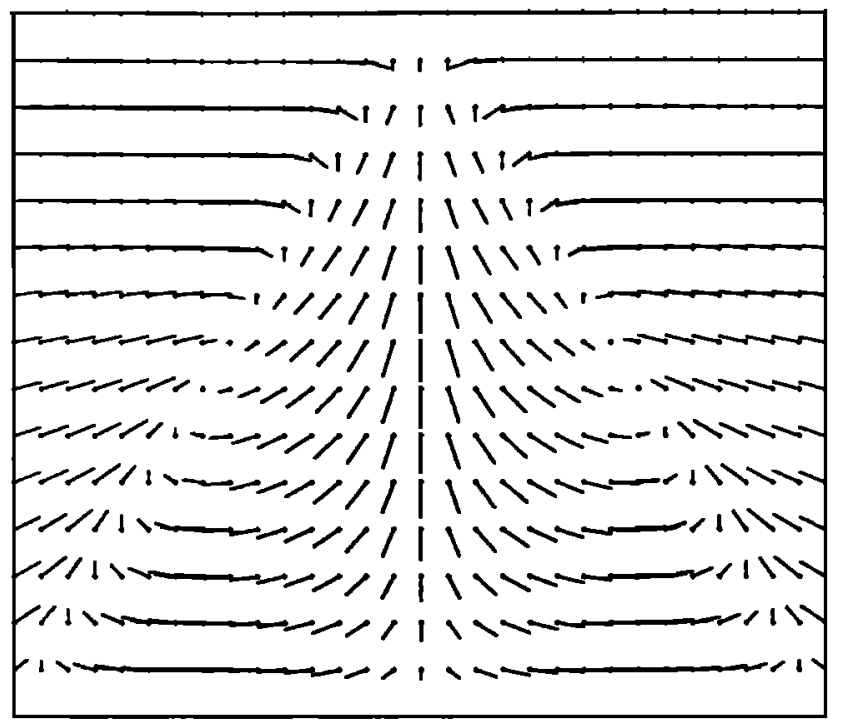

Fig. 1. Phase space trajectories for model 1 with zero forcing. The abscissa is $x$ from -1 to 1 ; the ordinate is $y$ from zero to 1 . At each location the straight line points downstream from the dot. The left and right parts of the figure correspond to dust predominantly in the south and north, respectively. The top and bottom parts of the figure correspond to large and small amounts of dust, respectively. The separatrix $x=0$ terminates at the unstable equilibrium point $x$ $=0, y=0$. Stable equilibrium points occur at $x= \pm 0.5, y=0.5$. The circulation is clockwise for $x<0$ and is counterclockwise for $x$ $>0$.

year, which is consistent with the 1977 storms observed by Viking [Zurek, 1982]. We ruthlessly shrink the problem down to two nonlinear time-dependent differential equations with seasonal forcing. If all the solutions to these equations were periodic with a 1-year period, then we would have to look for hidden microphysical variables or accept the idea of a delicately balanced climate. But if the solutions exhibited multiyear periodicities or aperiodic (chaotic) behavior for a wide range of parameters, then the global climate system might be the culprit. The next step would be to search for interannual variability in more comprehensive global models

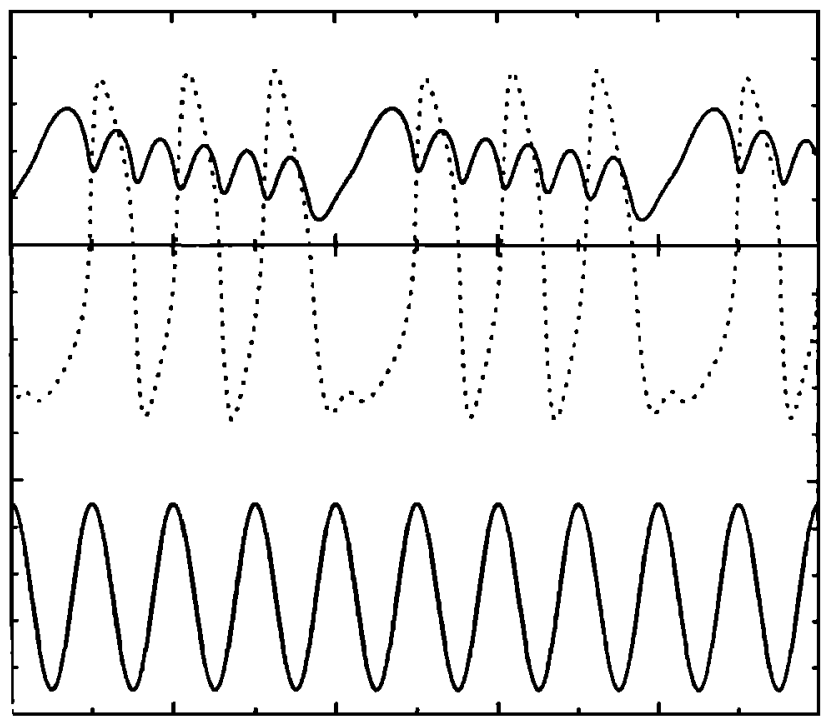

Fig. 3a. Time series of $x(t), y(t), f(t)$ for model 1 with $F=0.8$ and $\varepsilon=0$. The initial conditions are $x=-0.3, y=0.5$, and the integration step is $1 / 200$ of the forcing period. The dotted curve is $x(t)$; the upper solid curve is $y(t)$; the horizontal line is the zero of $x(t)$ and $y(t)$, and the distance between the horizontal line and the top of the box is one unit. The lower solid line is $f(t)$ with arbitrary amplitude scale. The tick marks along the abscissa are 1 year apart and are located at the times when $f(t)$ has its maximum value.

of Martian climate. The same philosophy underlies the attempts of Lorenz [1963], Vallis [1986], and Pedlosky [1987] to understand irregular cycles in the Earth's atmosphere and oceans.

\section{Eouations}

Let $n$ be the amount of dust in the atmosphere in the northern hemisphere, and $s$ be the corresponding amount in the southern hemisphere. The model computes the time derivatives $d n / d t$ and $d s / d t$ from the current values $n(t)$,

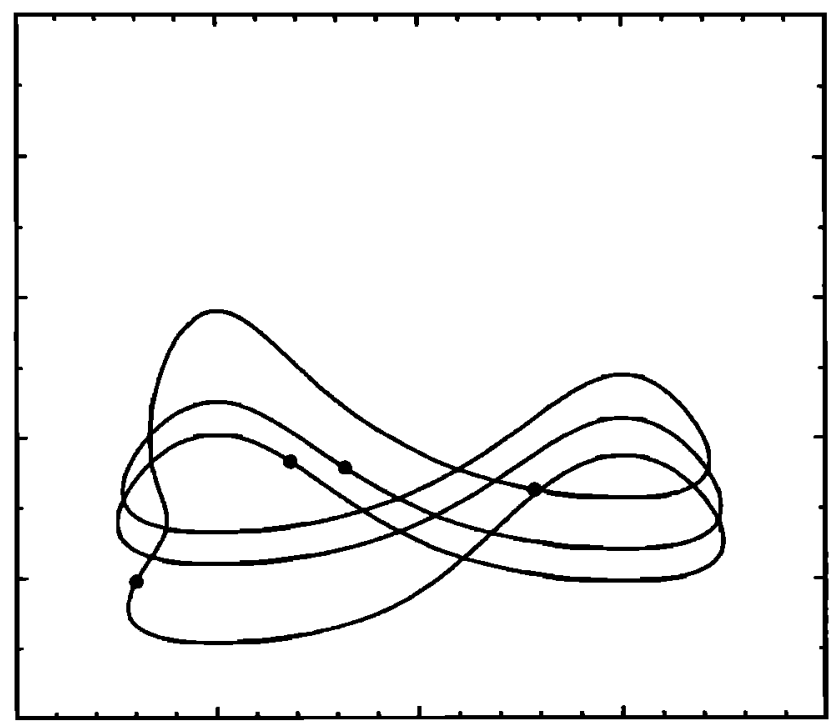

Fig. $3 b$. Phase space trajectory corresponding to Figure $3 a$. The scales are the same as in Figures 1 and 2. The dots show the state of the system on successive years at maximum positive forcing $\left(\phi=0^{\circ}\right)$.

Fig. 2. Same as Figure 1 but for model 2. 


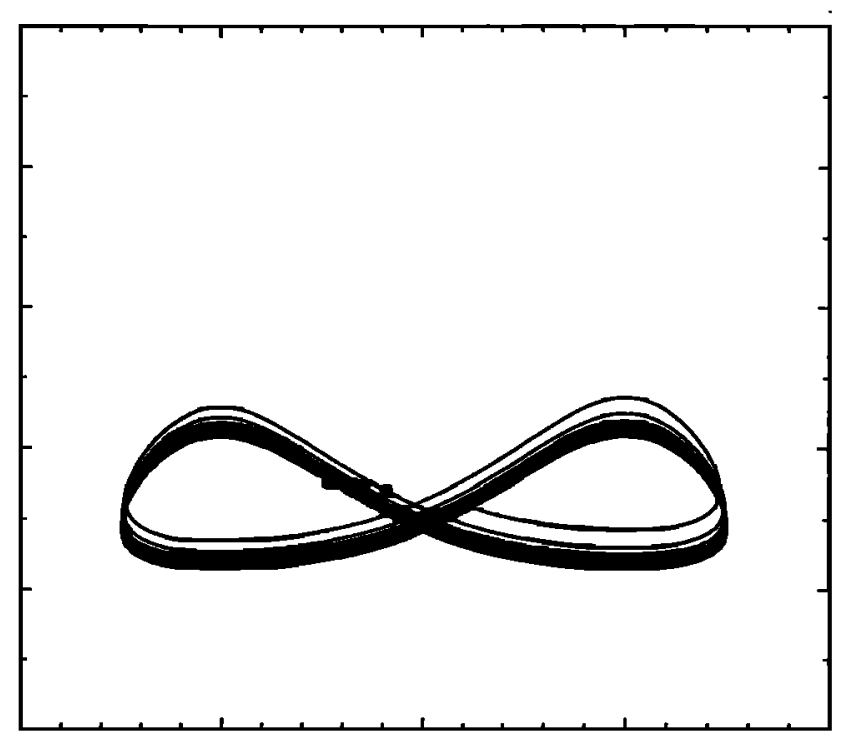

Fig. 4. Phase space trajectory for model 1 with $\varepsilon=0$ and $F=$ 0.85 . One hundred years are shown. Although the overall pattern is the same from year to year, the trajectories do not repeat exactly. The system is aperiodic.

$s(t)$, and a seasonal forcing function $f(t)$. The forcing is known, with period equal to the Mars year, so the model can be integrated forward from initial conditions $n(0), s(0)$ at time $t=0$. The model describes the following processes: atmospheric dust absorbs sunlight and heats the air. Air rises in the hemisphere where the heating is greatest and sinks in the opposite hemisphere, giving rise to a cross-equatorial, zonally symmetric circulation. The winds associated with this circulation pick up dust and advect it into the hemisphere where air is rising. Dust is carried to high altitudes and spreads into the opposite hemisphere, thereby removing the drive for the circulation. Dust falls out, and the amount of dust in the atmosphere decays toward the background level.

In each hemisphere the heating rate $R$ is assumed to be proportional to the amount of atmospheric dust. The strength of the cross-equatorial Hadley cell depends on the difference in heating rates between the northern and southern hemispheres. First, consider equinox conditions, when the sun is overhead at the equator; seasonal forcing will be considered later. At equinox the difference in heating rates is proportional to $n-s$. The rate $m$ at which air is exchanged between hemispheres is therefore given by $c(n-s)$, where $c$ is a positive constant. This relation is consistent with the dynamics of steady, zonally symmetric circulations [e.g., Holton, 1992, equation 10.15] when the eddy heat and momentum fluxes are negligible. Positive and negative values of $m$ correspond to air rising in the northern and southern hemispheres, respectively.

If the dust is concentrated close to the surface, the net effect of the cross-equatorial Hadley cell is to bring dust into the hemisphere where the air is rising. We define $(1+\alpha) / 2$ as the fraction of dust in the lower half of the atmospheric column and $(1-\alpha) / 2$ as the fraction of dust in the upper half. Note that $\alpha$ is 1 when all the dust is in the lower half, $\alpha$ is zero when the dust is evenly distributed, and $\alpha$ is negative when more of the dust is in the upper half of the atmospheric column. When air is rising in the northern hemisphere $(m>$
$0), d n / d t$ includes a term $s|m|(1+\alpha) / 2$, representing air entering the northern hemisphere from the southern hemisphere, and a term $(-n)|m|(1-\alpha) / 2$, representing air leaving the northern hemisphere into the southern hemisphere. The same terms appear with opposite signs in the expressions for $d s / d t$. When air is rising in the southern hemisphere $(m<0)$, the same expressions apply but with $\alpha$ replaced by $-\alpha$. The total dust in the atmosphere $n+s$ does not change as a result of these advections, but the dust asymmetry $n-s$ does change. With $m=c(n-s)$, the equations are

$$
\begin{aligned}
& \frac{d n}{d t}=+m \alpha\left(\frac{n+s}{2}\right)-|m|\left(\frac{n-s}{2}\right)+\frac{g|m|}{2}+\frac{b}{2}-\frac{n}{\tau}, \\
& \frac{d s}{d t}=-m \alpha\left(\frac{n+s}{2}\right)+|m|\left(\frac{n-s}{2}\right)+\frac{g|m|}{2}+\frac{b}{2}-\frac{s}{\tau}
\end{aligned}
$$

The first two terms in each equation describe interhemispheric advections. The $g|m| / 2$ term describes raising of dust from the surface by winds that are part of the dust-driven circulation; this term is proportional to the magnitude of $m$. The $b / 2$ term describes raising of dust by background winds that are independent of the dust-driven circulation. The $n / \tau$ and $s / \tau$ terms represent the fallout of dust onto the surface. The quantities $b, c, g$, and $\tau$ are constants, while the quantity $\alpha$ may depend on $n$ and $s$. We will choose $\alpha$ to be a decreasing function of atmospheric dust amount, with maximum value equal to unity.

We now rewrite the equations in dimensionless form. When the circulation is zero $(m=0, n-s=0)$ the total dust amount $n+s$ approaches the background level $b \tau$. We therefore define dimensionless variables $u$ and $v$ as the dust asymmetry and total dust amount above background, respectively, scaled by $b \tau$ :

$$
u=\left(\frac{n-s}{b \tau}\right) \quad v=\left(\frac{n+s-b \tau}{b \tau}\right) .
$$

Time is scaled by $\tau$, and the equations for the time derivatives $d u / d t$ and $d v / d t$ are obtained by adding and subtracting (1) and (2) after substituting $m=c(n-s)$.

$$
\begin{gathered}
\frac{d u}{d t}=\beta u(\alpha v+\alpha-|u|)-u, \\
\frac{d v}{d t}=\gamma|u|-v .
\end{gathered}
$$

Here $\beta$ and $\gamma$ are dimensionless constants,

$$
\beta=b c \tau^{2} \quad \gamma=g c \tau,
$$

and $\alpha$ is a dimensionless function of $u$ and $v$, as yet unspecified. We define $x$ as $u / v$ and derive its time derivative from (4) and (5):

$$
\frac{d x}{d t}=x[\alpha \beta(v+1)-(\beta v+\gamma)|x|] .
$$

When $x$ is equal to zero, the dust is evenly distributed between the northern and southern hemispheres. When $x$ is 


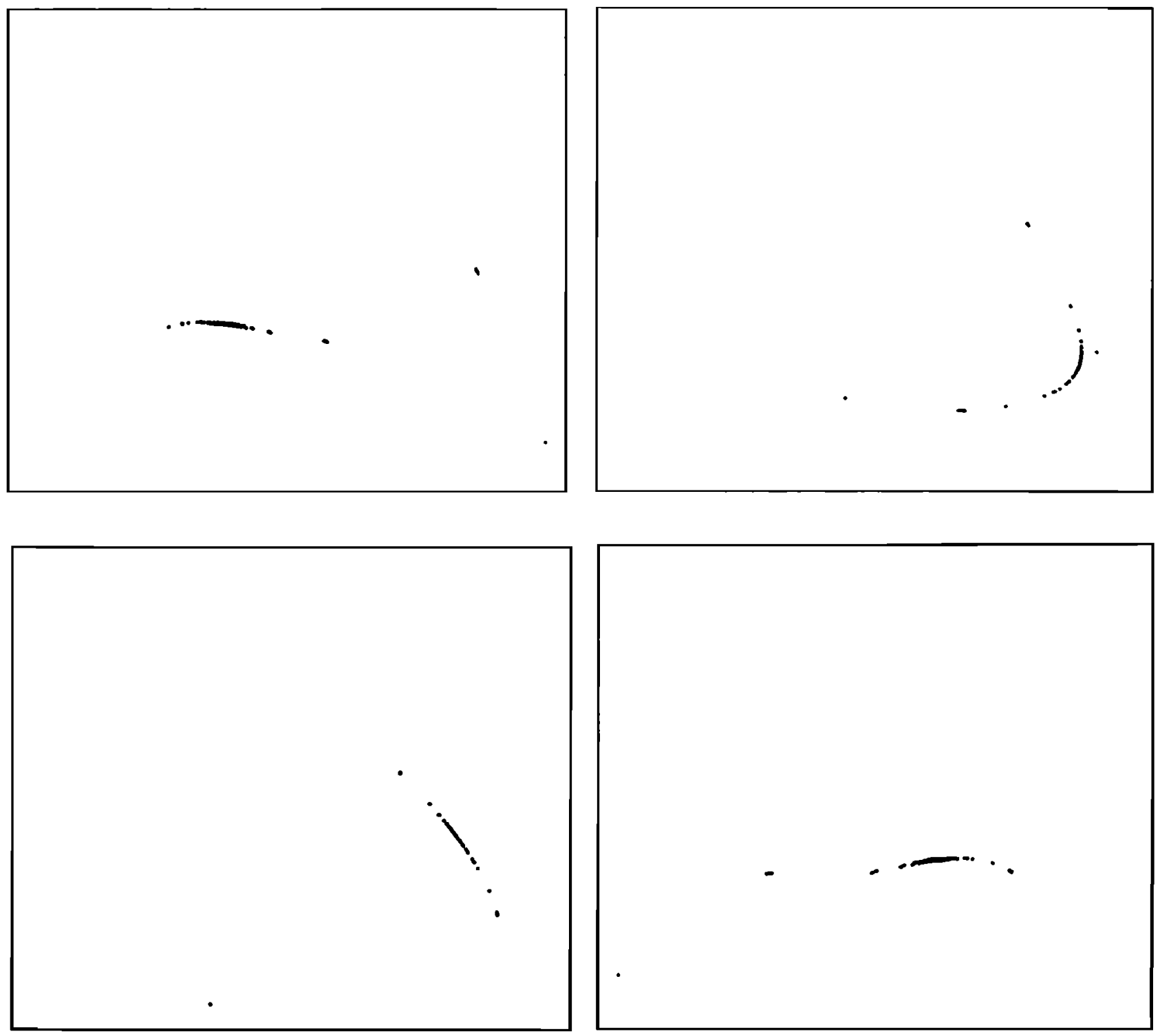

Fig. 5. Time slices in phase space from a 250-year run of model 1 for the same values of $\varepsilon$ and $F$ as Figure 4. The scales are the same as in Figures 1 and 2. Within each panel the dots record the state of the system at different years for the same phase $\phi$. The value of $\phi$ is $0^{\circ}$ in the upper left panel, and increases by $60^{\circ}$ from left to right and then down. Slices at $\phi=240^{\circ}$ and $\phi=300^{\circ}$ may be obtained by noting that a phase shift of $180^{\circ}$ is equivalent to a reflection about the line $x=0$. The initial conditions are $x=-0.3$ and $y=0.5$. At each phase the dots form a line in phase space, so the system is aperiodic.

greater than zero, more of the dust is in the northern hemisphere. When $x$ is less than zero, more of the dust is in the southern hemisphere. We also define $y$ as $v /(v+1)$, so that $y$ is confined to the range $0<y<1$. When $y$ is small, the total dust amount is close to the background level, and when $y$ is close to 1 , the total dust amount is large compared to the background level.

The model is still rich with possibilities, and we consider only two. In both cases we choose $\beta=\gamma=2$. The choice $\gamma$ $=2$ makes the time scale for raising dust the same as the time scale for dust fallout. Equation (5) becomes

$$
\frac{d v}{d t}=v(2|x|-1) .
$$

When $|x|<1 / 2$, the dimensionless total dust amount $v$ decays exponentially, but when $|x|>1 / 2, v$ grows. The changeover represents the interplay between fallout of dust and raising of dust by the dust-driven circulation. The strength of the circulation is proportional to $|n-s|$ and therefore to $|x|$. Fallout prevails when the dust is evenly distributed between the northern and southern hemispheres and the circulation is weak. Raising prevails when more dust is in one hemisphere and the circulation is strong. Increasing the value of $\gamma$ increases the region in phase space where dust can grow.

The choice $\beta=2$ guarantees that $x$ never goes outside the range $-1 \leq x \leq 1$, according to (7) with $\gamma=2$ and $|\alpha| \leq 1$. This is a convenient choice because the phase space of the model is then $-1 \leq x \leq 1,0 \leq y \leq 1$.

In model 1 we choose $\alpha=1 /(v+1)$. This is equivalent to $\alpha=1-y$, which has the dust concentrated in the lower half of the atmosphere $(\alpha=1)$ when the total dust amount is 
small $(v \ll 1)$, and has the dust evenly distributed with altitude $(\alpha \rightarrow 0)$ when the total dust amount is large $(v \gg 1)$. Contours of $\alpha$ are horizontal lines in the $x-y$ plane with $\alpha$ decreasing upward.

We choose a seasonal forcing function of the form $(1-$ $|x|) f(t)$ in the equation for $d x / d t$. Here $f(t)$ is given by $F$ $\cos \Omega t$, where $F$ is the dimensionless forcing amplitude and $\Omega$ is the dimensionless forcing frequency, given by $2 \pi \tau /$ (Mars year). The factor $(1-|x|)$ ensures that the forcing can never drive the system outside the domain $-1<x<1$. The fact that $f(t)$ changes sign every half year simulates the alternate heating of the northern and southern hemispheres. In terms of $x$ and $y$ the equations are

$$
\begin{gathered}
\frac{d x}{d t}=2 x\left[1-\frac{|x|}{(1-y)}\right]+(1-|x|) f, \\
\frac{d y}{d t}=y(1-y)(2|x|-1) .
\end{gathered}
$$

When the forcing is zero, model 1 has stable equilibria $(d x / d t$ $=d y / d t=0$ ) at the points $x= \pm 1 / 2, y=1 / 2$, toward which the system decays with dimensionless time constant unity (decay time $\tau$ ). Figure 1 shows the trajectories in phase space with $f=0$. The circulation is clockwise in the left half of the figure and is counterclockwise in the right half of the figure. The trajectories are not closed; rather, they converge toward the stable equilibrium points. Model 1 therefore describes a dissipative system.

Model 2 was deliberately chosen to give closed trajectories in phase space. Without forcing, model 2 is a Hamiltonian system: the phase space velocities $d x / d t$ and $d y / d t$ are derivable from the gradient of a stream function, which is the Hamiltonian. We choose the same form of the forcing function as in model 1 . In terms of $x$ and $y$, the equations for model 2 are

$$
\frac{d x}{d t}=\frac{\partial h}{\partial y}+(1-|x|) f=(1-|x|)[(1-2 y) x+f],
$$

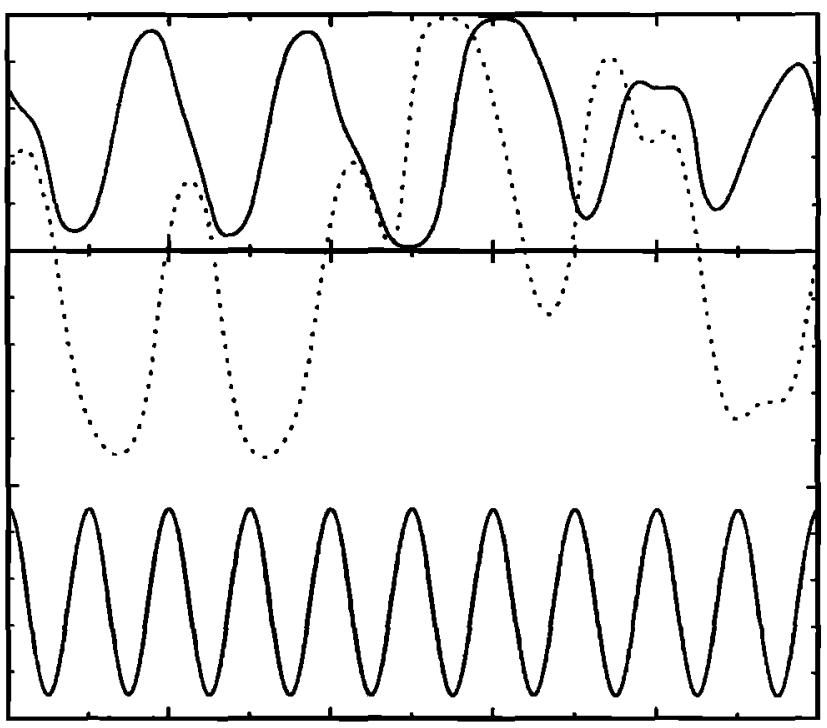

Fig. 6a. Same as Figure $3 a$, but for model 2 with $F=0.25$ and $\varepsilon$ $=0$. The initial conditions are $x=-0.3$ and $y=0.5$.

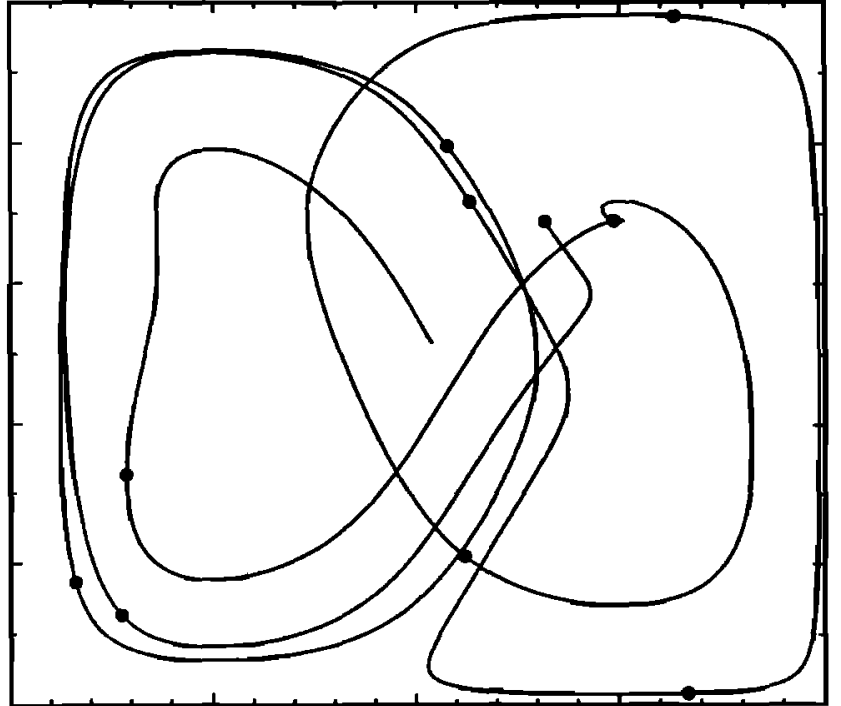

Fig. 6b. Phase space trajectory corresponding to Figure $6 a$. The dots show the state of the system at a phase $\phi=0^{\circ}$. The motion along the trajectory is generally clockwise in the left half of the figure and counterclockwise in the right half of the figure.

$$
\frac{d y}{d t}=-\frac{\partial h}{\partial x}=y(1-y)(2|x|-1),
$$

where the Hamiltonian $h$ is given by

$$
h=x(1-|x|) y(1-y) \text {. }
$$

This Hamiltonian is algebraically simple and has the desirable property that the phase space boundary $(y=0, y=1$, $|x|=1$ ) is a streamline, i.e., $h=0$. Requiring consistency between these equations and (7)-(8) yields the $\alpha(x, y)$ for model 2:

$$
2 \alpha=1+|x|+(1-|x|)\left(2 y^{2}-3 y\right) .
$$

The $\alpha$ contours form an elliptical bull's eye pattern centered at $x=0, y=3 / 4$, where $\alpha$ has its minimum value of $-1 / 16$. At smaller values of $y, \alpha$ is a decreasing function of atmospheric dust amount, as with model 1 . The models differ, however, in the location of the $\alpha=1$ contour. In model 1 the $\alpha=1$ contour (dust confined to the lower half of the atmosphere) occurs where $y=0$ (low dust amount). In model 2, the $\alpha=1$ contour occurs where $|x|=1$ (dust confined to either the northern or southern hemisphere). The former seems more realistic than the latter, but both models oversimplify.

When the forcing is zero, model 2 has stable equilibria at the points $x= \pm 1 / 2, y=1 / 2$, around which the system circulates with dimensionless frequency $1 / 2$ (period $4 \pi \tau$ ). Figure 2 shows the trajectories in phase space with $f=0$. The direction of circulation is the same as for model 1 . Because each trajectory closes on itself, model 2 describes a dissipationless system.

\section{RESULTS}

The goal is to see whether time-dependent forcing with an annual period can lead to interannual variability. Both models 1 and 2 have a separatrix at $x=0$ that terminates at 

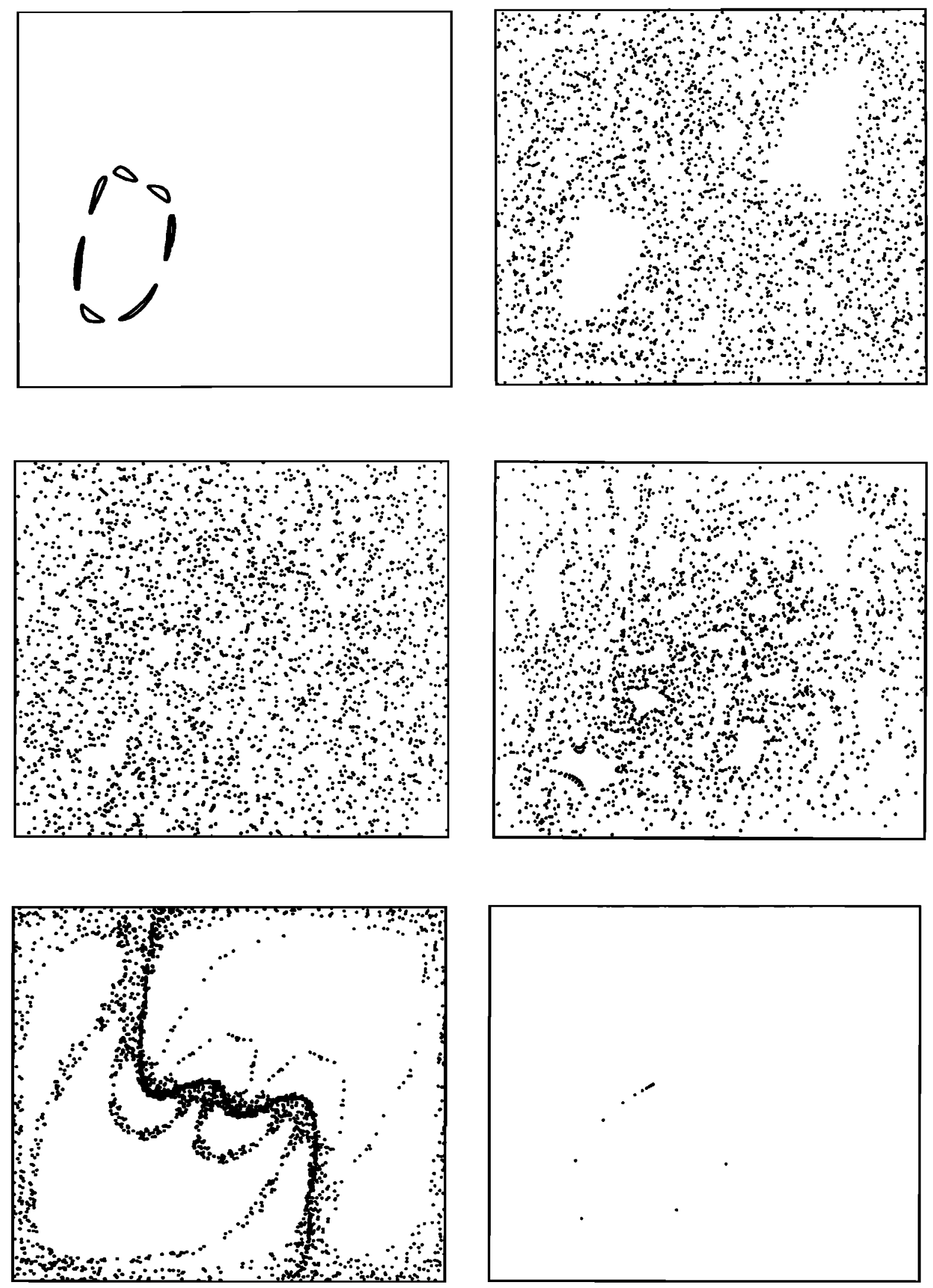

Fig. 7. Time slices in phase space from a series of 2500-year runs of model 2 for $\varepsilon=0$ and various $F: 0.10$ (upper left), 0.13 (upper right), 0.25 (center left), 0.50 (center right), 0.55 (lower left), and 0.70 (lower right). The initial conditions are $x=-0.3, y=0.5$. The dots record the state of the system in different years at the same phase $(\phi=$ $180^{\circ}$ ). As with model 1 (Figures 4 and 5), the system is aperiodic, but here the dots fill areas of phase space. 
the unstable equilibrium point $x=0, y=0$. The timedependent forcing can move the system back and forth across the separatrix, from the domain of the steady northern hemisphere dust storm $(x=1 / 2, y=1 / 2)$ to the domain of the steady southern hemisphere dust storm $(x=-1 / 2, y$ $=1 / 2$ ) and back. With periodic forcing, the response of the system could be either periodic or aperiodic (chaotic). The response period could be equal to the forcing period, which is the Mars year, or to any integer multiple of it. The fact that model 2 is a Hamiltonian system with a separatrix and time-dependent forcing guarantees that it will have chaotic trajectories. Many of these possibilities involve interannual variability. The question is, how pervasive is this variability and what are its properties?

In the results that follow, the dust storm decay time $\tau$ is taken to be $1 / 10$ of the Mars year. With $\tau$ the unit of time, the dimensionless forcing frequency $\Omega$ is therefore $2 \pi / 10$. The forcing function is either $f(t)=F \cos \Omega t$, or else

$$
f(t)=F[(\cos \Omega t)(1-2 \varepsilon \cos \Omega t)+\varepsilon] .
$$

With $\varepsilon=0.093$, (15) crudely simulates the eccentricity of the Martian orbit. We define the phase $\phi$ of the forcing as $360^{\circ}$ times the remainder of the quotient $\Omega t$ divided by $2 \pi$. Since the forcing appears in the equation for $d x / d t$, maximum positive forcing $\left(\phi=0^{\circ}\right)$ corresponds to northern summer solstice $\left(L s=90^{\circ}\right)$. Thus $L s=\phi+90^{\circ}$. Equation (15) tends to produce short, intense summers in the south ( $L s=$ $270^{\circ}, \phi=180^{\circ}$ ) and long, mild summers in the north. This is consistent with the Mars orbit, where perihelion passage occurs at $L s \approx 250^{\circ}$ corresponding to $\phi \approx 160^{\circ}$.

The forcing amplitude $F$ can be regarded as the steady state value of $x$ that would result if the circulation were zero and dust merely settled out of the atmosphere with time constant $\tau$. For finite $F$, the factor $(1-|x|)$ in (9) and (11) reduces the response amplitude. A value of $F$ of the order of 0.5 or 1.0 therefore represents a reasonable seasonal amplitude for Mars.

Figure $3 a$ shows the time series $x(t), y(t), f(t)$ for model 1 with a forcing amplitude $F=0.8$ and eccentricity $\varepsilon=0$. Figure $3 b$ shows the corresponding trajectory in phase space. The dots in Figure $3 b$ show the state of the system on successive years when $\phi=0^{\circ}$, corresponding to $L s=90^{\circ}$. The direction of motion along the trajectory is consistent with the unforced motion shown in Figure 1, clockwise in the left half of the figure and counterclockwise in the right half of the figure. The trajectory is periodic with a period of 4 years and is asymmetric about $x=0$ despite the symmetry of the forcing. Some values of $F$ give symmetric trajectories when $\varepsilon=0$, and other values give asymmetric trajectories with different periods. For certain other initial conditions, the system shown in Figure 3 settles into a trajectory that is the mirror image of this one reflected about the line $x=0$. The time constant for decay into one of these trajectories is about 1 year.

The forcing amplitude in Figure 3 is an intermediate value. For values of $F$ less than 0.6 there are two trajectories, each with a period of 1 year. One trajectory oscillates about the equilibrium point $x=1 / 2, y=1 / 2$, and the other oscillates about the equilibrium point $x=-1 / 2, y=1 / 2$. For values of $F$ greater than 0.85 there is only one trajectory; its period is 1 year, and the state of the system loops back and forth between the two equilibrium points in a figure-eight pattern. For intermediate values of $F$, multiyear periods are possible.

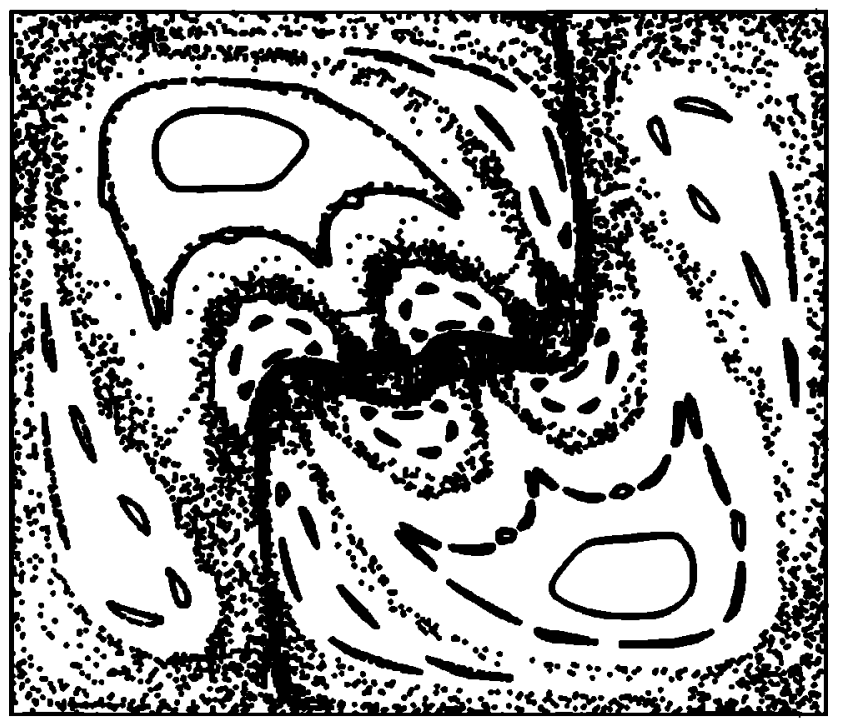

Fig. 8. Model 2 with multiple initial conditions. The coordinates of the plot and the values of $\varepsilon$ and $F$ are the same as in the lower left panel of Figure 7 . The phase here is $0^{\circ}$, so the trajectory of the Figure 7 panel appears as its own mirror image reflected about the line $x=0$.

Since $y$ is $v /(v+1)$, where $v$ is proportional to the total dust amount, the ratio of the largest dust storm in Figure 3 (largest maximum of $y$ ) to the smallest dust storm (smallest maximum of $y$ ) is about 2.25 to 1 . The largest dust storm in Figure 3 occurs in the south, just after summer solstice in the southern hemisphere. Both northern and southern hemisphere storms are equally likely in this model, when $\varepsilon=0$. In general, the maxima of $y$ in each hemisphere occur during the summer season of that hemisphere $\left(0<\phi<90^{\circ}\right.$ in the north and $180<\phi<270^{\circ}$ in the south). Adding eccentricity to the forcing (15) adds a small asymmetry to trajectories that were symmetric before, but does not change the general amplitude or phase relations illustrated in Figure 3.

Model 1 becomes aperiodic (chaotic) in a narrow band of forcing amplitudes centered at $F=0.85$. An example is shown in Figure 4. The trajectory spans 100 years beginning 900 years after the initial start-up. Although the general shape of the pattern does not change from year to year, the phase of the response relative to the forcing (the location of the dot on each trajectory) is seen to wander. Figure 5 shows a 250-year history sampled (sliced) at four different times of the year $\left(\phi=0^{\circ}, 60^{\circ}, 120^{\circ}, 180^{\circ}\right)$. Within each panel, the state of the system during each year is represented by a dot. A trajectory with a period of $N$ years would appear as $N$ dots. An aperiodic trajectory, or one with a period that is incommensurate with the forcing period, would appear as a continuous line or filled area. Whether this trajectory is periodic with a very long period (large $N$ ), or is truly aperiodic, is hard to say. In either case the state of the system (location of the dot) at a given time of year (value of $\phi$ ) can take on many values.

Model 2 shows much more spectacular examples of aperiodic trajectories. Figures $6 a$ and $6 b$ show a time series and a phase space trajectory of a model 2 integration with $F=$ 0.25 and $\varepsilon=0$, respectively. Only a portion of the integration is shown, long after any initial transients have died away, but the pattern never repeats. Figure 7 shows time 

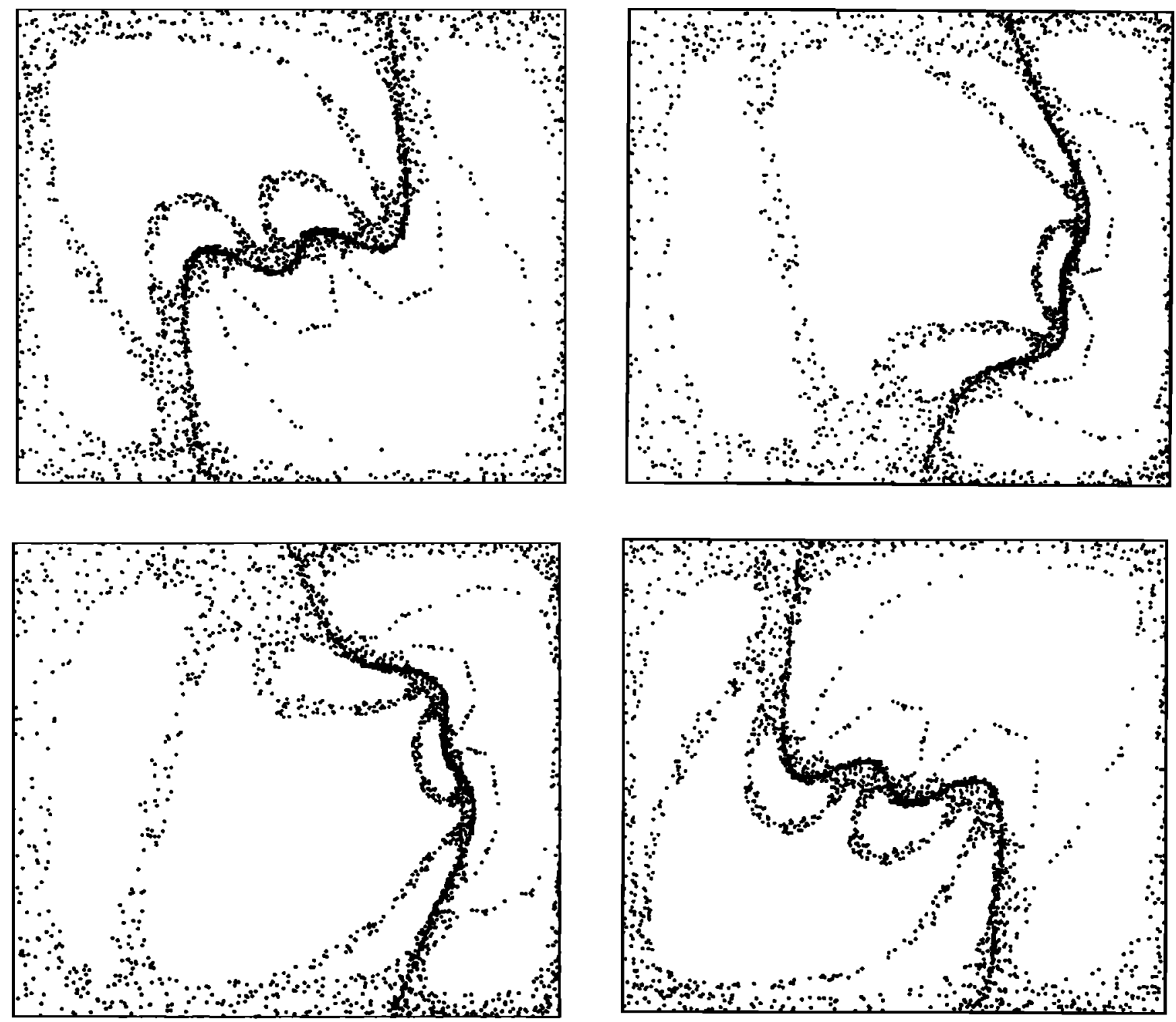

Fig. 9. Time slices in phase space from a 2500-year run of model 2 for $\varepsilon=0$ and $F=0.55$, the same parameter values used in Figure 8 and in the lower left panel of Figure 7. The values of $\phi$ range from $0^{\circ}$ to $180^{\circ}$ (increasing by $60^{\circ}$ from left to right and then down). The initial conditions are $x=-0.3, y=0.5$. The cases $\phi=240^{\circ}$ and $\phi=300^{\circ}$ may be obtained by reflecting the cases $\phi=60^{\circ}$ and $\phi=120^{\circ}$ about the line $x=0$.

slices, similar to those of Figure 5, from a series of 2500-year runs of model 2 ( $\varepsilon=0$, different values of $F$ ), in which the state of the system is recorded once each year (at $\phi=180^{\circ}$ ) as a single dot in the $x-y$ plane. Except for the change in phase (from $\phi=0^{\circ}$ to $\phi=180^{\circ}$ ), the dots have the same significance as in Figure $6 b$. The fact that the dots fill a finite area means that the system is chaotic for these values of parameters.

Model 2 is not always chaotic. As shown in Figure 2, all of the trajectories of the unforced system are periodic. Those closest to the stable equilibrium points at $|x|=1 / 2, y=1 / 2$ have the shortest periods, one-fifth of the Mars year. Those outside have longer periods, with two infinite-period trajectories circulating in opposite directions down the line $x=0$ and around the outer boundaries. Both the long-period and the short-period trajectories become aperiodic when forcing is added, as the upper two panels of Figure 7 illustrate. All six panels were started at the same initial condition, $x=$ $-0.3, y=0.5$. For $F=0.10$ (upper left panel), the initial point is inside the domain of the short-period trajectories. These circulate around the equilibrium points, as in the unforced system, but their domain shrinks as $F$ increases. For $F=0.13$ (upper right panel) the initial point is in the domain of the long-period trajectories, which fill most of phase space.

In the center left panel $(F=0.25)$ of Figure 7 the trajectory appears to fill all of the phase space. All states of the system are allowed at any time of the year. But for $F=$ 0.50 (center right panel) there are islands sheltering different trajectories, which are confined in phase space. Increasing $F$ to 0.55 produces the strange attractor shown in the lower left panel. The white areas of that panel are occupied by different trajectories, which are reached by different initial conditions. Some of these trajectories are shown in Figure 8, 


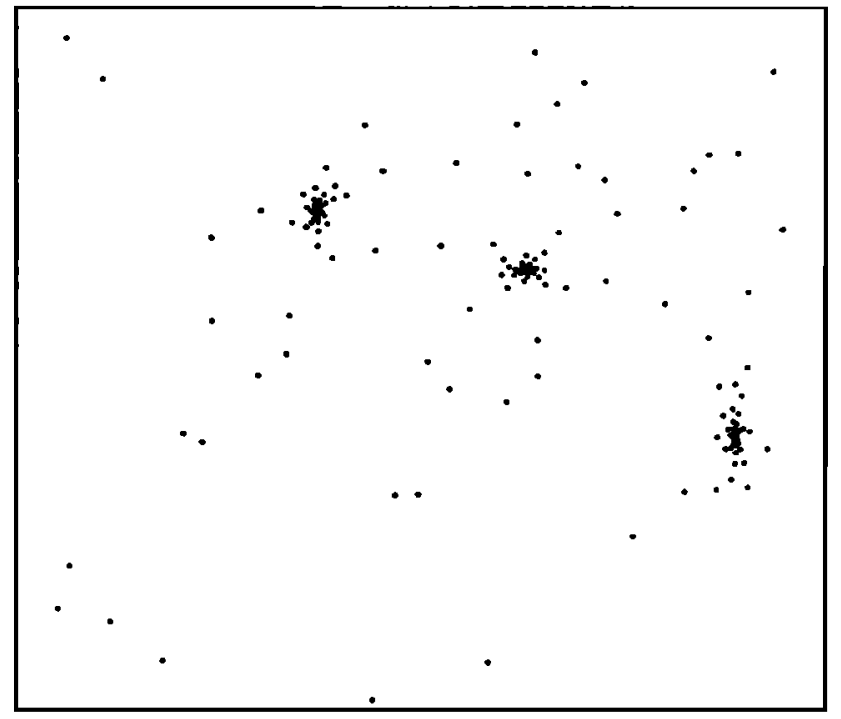

Fig. 10a. Time slices in phase space from a 2500-year run of model 2 with $\varepsilon=0.093$ and $F=0.50$. The initial conditions are $x=$ $-0.3, y=0.5$, and the phase of the slice is $\phi=180^{\circ}$.

which also has $F=0.55$. The phase $\left(\phi=0^{\circ}\right)$ differs by $180^{\circ}$ from that of Figure 7, so the trajectory in the lower left panel of Figure 7 appears reflected about the line $x=0$ in Figure 8. The spiral pattern of dots in the lower right panel $(F=$ 0.7 ) represents a transient that decays to a periodic trajectory with a period of one year.

For comparison with dust storms on Mars, an important question concerns the phasing (seasonality) of the major events (large values of $y$ ). Figure 9 shows time slices for $F=$ $0.55, \varepsilon=0$, at a range of phases from $0^{\circ}$ to $180^{\circ}$. The lower right panel $\left(\phi=180^{\circ}\right)$ of Figure 9 corresponds to the lower left panel of Figure 7. The upper left panel of Figure 9 is for northern summer solstice. The cluster of points moves into the summer hemisphere (upper right panel), circulating around counterclockwise in the right half of the figure (lower left panel), and then back to the equator at southern summer

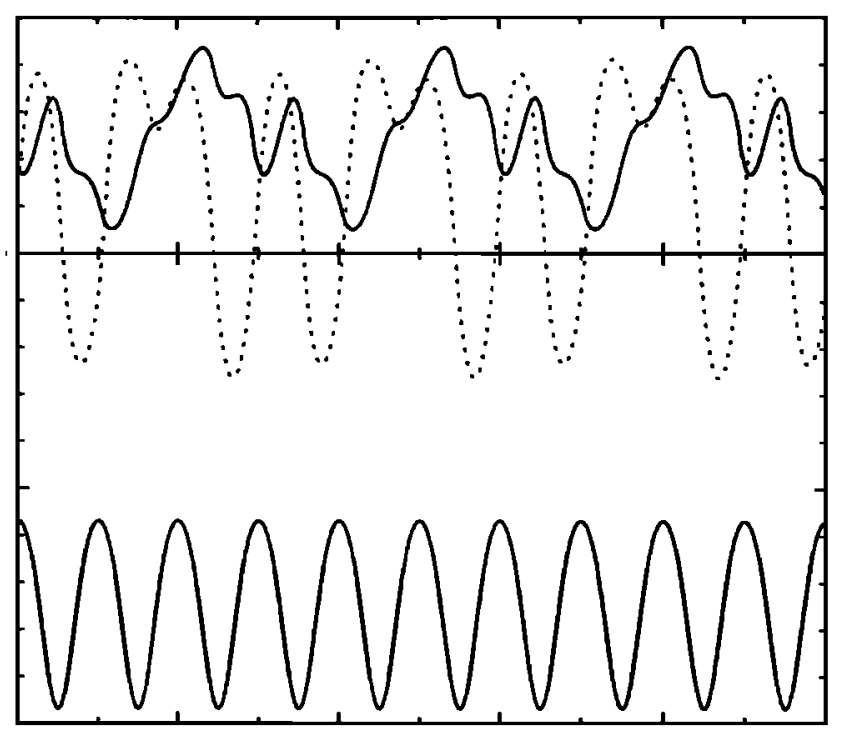

Fig. 10b. Time series corresponding to a short section of the run in Figure $10 a$ after the transient has died away.

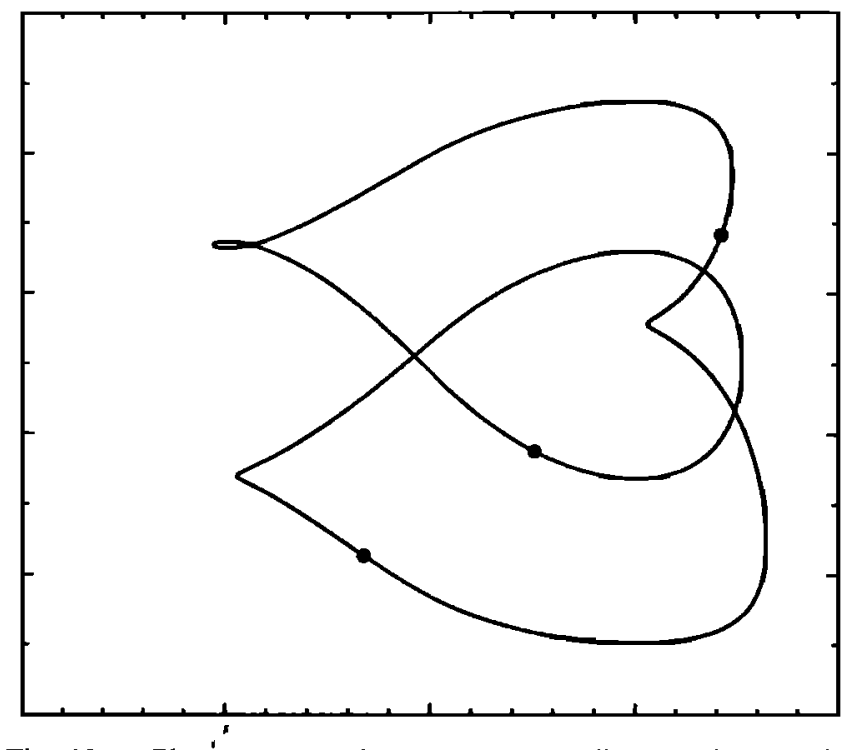

Fig. 10c. Phase space trajectory corresponding to Figure $10 b$ The dots show the state of the system at $\phi=0^{\circ}$.

solstice (lower right panel). The symmetry of (11)-(13) under the transformations $x \rightarrow-x, y \rightarrow 1-y$ means that the mirror image of each pattern appears during the second half of the cycle. According to the figure, the dust is most likely to be concentrated in one hemisphere at the end of summer, e.g., between the upper right and lower left panels.

The trajectory shown in Figure 9 is just one of many, as illustrated in Figure 8. The other trajectories occupy the white spaces in Figure 9, and these white spaces also circulate counterclockwise in the right half of the figure. Some of these white spaces reach large amplitude (large values of $y$ ) and large hemispheric asymmetry (large values of $x$ ) at different phases in the seasonal cycle than the trajectory of Figure 9. Because model 2 is nondissipative, the trajectories do not cross. The system remembers its initial conditions for all time. Even though the region with the most dots shifts from one hemisphere to the other, every corner of phase space has dots in it. Large dust storms (large values of $y$ ) can occur at any season, according to this figure.

In general, changing the eccentricity $\varepsilon$ from 0 to 0.093 has little effect on the trajectories. However, when the initial point is near a boundary between trajectories of different types, then that particular solution changes dramatically. In the top two panels of Figure 7 we saw the effect of changing $F$ from 0.10 to 0.13 while holding the initial condition constant. In Figure 10 we see the effect of changing $\varepsilon$. The forcing $(F=0.50)$ and the initial condition $(x=-0.3, y=$ $0.5)$ are the same as for the middle right panel of Figure 7, but the eccentricity is now 0.093 . Changing this parameter moves the trajectories so that the system is now confined to one of the white spaces in the center right panel of Figure 7. Instead of wandering around phase space, the system is periodic, as shown in Figure 10. Figure $10 a$ is a time slice at $\phi=180^{\circ}$, as in Figure 7, but the pattern shows a transient that decays to a trajectory that has a period of 3 years. The three concentrations of dots are located close to three islands in the center right panel of Figure 7. Figure $10 b$ shows the time series after the transient has died away, and Figure $10 c$ shows the periodic trajectory in phase space. The circulation in Figure 10c is counterclockwise. As in Figure 
$3 b$, the dots in Figure $10 c$ show the state of the system at $\phi$ $=0^{\circ}$.

The two maxima of $y$ occur in the northern hemisphere, according to Figure 10. The largest of these occurs at the beginning of northern fall, well after the time of maximum heating in the north. Mars has large dust storms that originate in the south during southern summer. However, a different initial condition would put the system on a trajectory that is almost (because $\varepsilon$ is nonzero) a mirror image (about $x=0$ ) of the one shown. Such a trajectory has a major dust storm (large $y$ ) in the southern hemisphere at the beginning of southern fall. Although other trajectories are lurking nearby, the general appearance of Figures 7-9 is not changed by setting $\varepsilon=0.093$.

\section{Conclusions}

The calculations demonstrate that interannual variability is possible in simple global dust storm models, even when the natural response time of the system is an order of magnitude shorter than the period of the forcing. Both models employ two predicted variables, representing the amount of atmospheric dust in the northern and southern hemispheres, respectively. Algebraic relations connect these two variables to the strength of the circulation, the vertical distribution of the dust, and the interaction with the surface. The dust storm decays when it spreads into both hemispheres. This spreading is controlled by a function $\alpha$, which describes how the vertical distribution of dust depends on the circulation. Model 1 uses a straightforward relation for $\alpha$ : the dust is concentrated close to the ground in a clear atmosphere and is more uniformly distributed in a dusty atmosphere. Model 2 has this same general feature, but the relation was specially chosen to give oscillating behavior in the absence of forcing.

For reasonable parameter settings, model 1 gives solutions that behave like Martian dust storms. Dust storms develop in the springtime hemisphere and reach their peak in the summer, which is more or less in accord with the observations. The intensity and timing may vary from one year to the next, but the patterns usually repeat after a few years. Some patterns repeat every year; others repeat after several years. A few patterns have such long periods that they are essentially aperiodic. Some patterns are asymmetric and favor one hemisphere over the other; these solutions come in pairs, so the overall symmetry between hemispheres is preserved. Adding orbital eccentricity to the forcing does not greatly alter this symmetry. The principal weakness of model 1 , when compared to Martian dust storms, is that most of the solutions are periodic. Mars dust storms do not seem to be periodic, but the statistics are poor. A more realistic model, with more variables, might be either more or less periodic depending on the strength of the annual forcing compared to the model's own internal variability.

Model 2 has the advantage that it gives solutions that are clearly aperiodic (chaotic). For many parameter settings, the patterns never repeat from year to year. Large-amplitude interannual variability is the norm for this model, in seeming agreement with the observations, although the observational statistics are not adequate to draw a firm conclusion. Dust storms can occur at all seasons according to this model. The most frequent occurrence is at the end of summer or beginning of fall, which is significantly later than what is observed. Dust storms are equally likely in the northern and southern hemispheres. The orbital eccentricity, as modeled, does little to alter this behavior. On Mars the dust storms develop in the south and reach their peak around southern summer solstice.

The main disadvantage of a simple model like this one is the need to parameterize important physical processes. Physically interpreting the model becomes a complex task, even though the mathematics is simple. We have not explored all of parameter space with the present model. The constants $\beta$ and $\gamma$ were chosen to divide phase space equally between regions where dust storms decay and regions where they grow. By adjusting these constants, it might be possible to simulate the difference between the rapid growth (10-20 days) and slow decay (60-80 days) of global dust storms. Differences in topography between the northern and southern hemispheres were not taken into account. By exploring the effect of topography on dust storms, it might be possible to simulate the tendency of dust storms to begin in the south. And by further adjusting $\alpha$, it might be possible to move the preferred time for major dust storms from the beginning of fall to the beginning of summer, where they are observed. But perhaps a better approach is to reduce the number of parameters by making the model more realistic, by exploring the large middle ground between the global general circulation models and models of the sort presented here.

It is hoped that this paper will stimulate further research, first, to study the statistics of Martian dust storms, and second, to look for multiyear periods and chaotic behavior in more realistic global models. Our plan is to build upward from the two-parameter model, in order to further test the hypothesis that the global circulation is the source of interannual variability. Exotic microphysical processes may be at work, but simple models of the global circulation have great unexplored potential for generating their own exotic behavior.

Acknowledgments. The authors thank Richard Zurek for offering helpful suggestions and for making his paper with L. J. Martin available in advance of publication. This research was supported by the Mars Observer Project and by the NASA Planetary Atmospheres Program under grant NAGW-1956.

\section{REFERENCES}

Gierasch, P. J., Martian dust storms, Rev. Geophys., 12, 730-734, 1974.

Gierasch, P. J., and R. M. Goody, A model of a Martian great dust storm, J. Atmos. Sci., 30, 169-179, 1973.

Haberle, R. M., Interannual variability of global dust storms on Mars, Science, 234, 459-461, 1986.

Haberle, R. M., C. B. Leovy, and J. B. Pollack, Some effects of global dust storms on the atmospheric circulation of Mars, Icarus, $50,322-367,1982$.

Haberle, R. M., J. B. Pollack, J. R. Barnes, R. W. Zurek, C. B. Leovy, J. R. Murphy, H. Lee, and J. Schaeffer, Mars atmospheric dynamics as simulated by the NASA Ames General Circulation Model, 1, The zonal mean circulation, J. Geophys. Res., 98, 3093-3123, 1993.

Holton, J. R., An Introduction to Dynamic Meteorology, 3rd ed., pp. 316-319, Academic, San Diego, Calif., 1992.

Leovy, C. B., R. W. Zurek, and J. B. Pollack, Mechanisms for Mars dust storms, J. Atmos. Sci., 30, 749-762, 1973.

Leovy, C. B., J. E. Tillman, W. R. Guest, and J. Barnes, Interannual variability of Martian weather, in Recent Advances in Planetary Meteorology, edited by G. E. Hunt, pp. 69-84, Cambridge University Press, New York, 1985. 
Lorenz, E. N., Deterministic nonperiodic flow, J. Atmos. Sci., 20, 130-141, 1963.

Martin, L. J., The major Martian yellow storm of 1971, Icarus, 22, 175-188, 1974a.

Martin, L. J., The major Martian dust storms of 1971 and 1973, lcarus, 23, 108-115, $1974 b$.

Martin, L. J., 1973 dust storm on Mars: Maps from hourly photographs, Icarus, 29, 363-380, 1976.

Martin, L. J., Clearing the Martian air: The troubled history of dust storms, Icarus, 57, 317-321, 1984

Murphy, J. R., R. M. Haberle, O. B. Toon, and J. B. Pollack, Martian global dust storms: Zonally symmetric numerical simulations including size-dependent particle transport, J. Geophys. Res., 98, 3197-3220, 1993.

Pedlosky, J., Geophysical Fluid Dynamics, 2nd ed., pp. 589-616, Springer-Verlag, New York, 1987.

Pollack, J. B., D. S. Colburn, F. M. Flasar, R. Kahn, C. E. Carlston, and D. Pidek, Properties and effects of dust particles suspended in the Martian atmosphere, J. Geophys. Res., 84, 2929-2945, 1979.

Pollack, J. B., R. M. Haberle, J. Schaeffer, and H. Lee, Simulations of the general circulation of the Martian atmosphere, 1, Polar processes, J. Geophys. Res., 95, 1447-1473, 1990.
Schneider, E. K., Martian great dust storms: Interpretive axially symmetric models, Icarus, 55, 302-331, 1983.

Vallis, G. K., El Nino: A chaotic dynamical system?, Science, 232, 243-245, 1986.

Zurek, R. W., Martian great dust storms: An update, Icarus, 50, 288-310, 1982.

Zurek, R. W., and L. J. Martin, Interannual variability of planetencircling dust storms on Mars, J. Geophys. Res., 98, 3247-3259, 1993.

Zurek, R. W., J. R. Barnes, R. M. Haberle, J. B. Pollack, J. E. Tillman, and C. B. Leovy, Dynamics of the atmosphere of Mars, in Mars, edited by H. H. Kieffer et al., pp. 835-933, University of Arizona Press, Tucson, 1992.

A. P. Ingersoll and J. R. Lyons, Division of Geological and Planetary Sciences, California Institute of Technology, Pasadena, CA 91125.

(Received February 3, 1992;

revised March 15, 1993;

accepted March 17, 1993.) 